\title{
Effect of Surface Cleanliness of Aluminum Substrates on Silicone Rubber Adhesion
}

\author{
L. Petersson, P. Meier ${ }^{*}$, X. Kornmann ${ }^{*}$, H. Hillborg ${ }^{\#}$ \\ ABB Corporate Research, Power Technology, SE-721 78 Västerås, Sweden, \\ *ABB Corporate Research, Insulation \& Polymer Technologies, CH-5405 Baden Dättwil, \\ Switzerland
}

${ }^{\#}$ Corresponding author: Tel: +46 21 323102, Fax: +46 21323212

E-mail address: henrik.hillborg@ se.abb.com 


\section{ABSTRACT}

The aim of the present work was to determine the minimum surface cleanliness of aluminum substrates required for good and reproducible silicone rubber adhesion. Aluminum substrates were prepared, ranging from 'contaminated' to different degrees of 'cleaned'. The surface energy of the substrates was determined by contact angle measurements. The surfaces were also compared using simplified methods, such as a wettability test or by the use of inks with known surface tension. Silicone rubber was then compression moulded onto the cleaned and primed substrates. The silicone rubber adhesion was then evaluated by lap-shear testing, before and after ageing. The ageing step consisted of immersion of samples in boiling water during $100 \mathrm{~h}$ for evaluating the hydrolytic stability of the interfaces. The failure modes after lap shear testing were determined using optical microscopy and scanning electron microscopy and were divided into three different categories: cohesive failure, adhesive failure or a mixture thereof. Energy Dispersive X-ray mapping was useful in clarifying the failure modes by determining the position of the primer, which contained Ti. It was concluded that in order to obtain a strong and stable interface, exhibiting mainly cohesive failure between the aluminum substrate and silicone rubber, the surface energy of the substrate before priming should be $>45 \mathrm{~mJ} \mathrm{~m}^{-2}$, including a polar component of $>10 \mathrm{~mJ} \mathrm{~m}^{-2}$. This corresponded to a hydrophobicity class of the substrate of $\geq 6$, according to IEC 62073 .

\section{INTRODUCTION}

Successful injection molding of polymers onto various substrates requires good and reproducible adhesion between the two materials. Due to the low surface energy of silicone rubber, around $20 \mathrm{~mJ} \mathrm{~m}^{-2}$, it is difficult to bond silicones to other materials ${ }^{1,2}$. Usually primers are needed ${ }^{3}$, often in combination with incorporation of functional groups, through copolymerization $^{4}$ or through surface treatment ${ }^{5,6}$. However, there are many other parameters to consider in an industrial process. Example of such parameters are the cleanliness, surface roughness and type of substrates, type of primer system, air humidity and temperature during application and activation of primer, time between primer application and subsequent injection moulding, heat treatment of primed substrates, etc. If any of such process 
parameters deviate this may lead to loss of adhesion. In order to evaluate adhesion of polymer layers onto solid surfaces, peel testing has shown to be a repeatable and quantitative method ${ }^{7}$. There have been several attempts to correlate the adhesion of polymeric materials with the chemical composition of the surface. Iqbal et al. used lap shear testing to evaluate the effect of the total surface energy on adhesion between plasma-treated polyetheretherketone ${ }^{8}$ or epoxy $^{9}$ onto fiber reinforced polyphenylene oxide. The adhesion improved as a result of increased surface roughness as well as increased surface energy. Dartevelle et al. evaluated the effectiveness of plasma-cleaning for improving the strength and durability of adhesively bonded aluminum joints ${ }^{10}$. Improvement in adhesion was correlated with low carbon concentration, determined by X-ray photoelectron spectroscopy, and increased surface energy, as indicated by low water contact angles.

It is also important to remember that surfaces usually consist of constituents that are different from the bulk material. For metals and alloys, the surfaces usually consist of oxides and absorbed gases ${ }^{11}$. For example, an oxide layer is formed on aluminum when exposed to ambient conditions. Depending on the climatic conditions, two different types can develop ${ }^{12,13}$, either a nearly pore-free amorphous aluminum oxide $\left(\mathrm{Al}_{2} \mathrm{O}_{3}\right)$, or a porous and hydrated layer with low crystalline content consisting of aluminum hydroxide $\left(\mathrm{Al}(\mathrm{OH})_{3}\right)$ and bayerite. If a polymer is moulded onto aluminum, the mechanically weaker porous oxide may result in bad adhesion when exposed to mechanical deformations. The failure will then propagate within the oxide layer, exposing the aluminum substrate, whereas other parts of the oxide and primer will remain on the polymer. Aluminum oxide layers can be removed chemically, for example by using an alkaline cleaning agent, before priming the substrate ${ }^{14,15}$.

The aim of the present work was to determine the minimum surface cleanliness of aluminum substrates required for good and reproducible silicone rubber adhesion. Aluminum substrates were cleaned using three different methods involving 1) ethanol, 2) ethanol and acetone or 3) an alkaline detergent. As comparison, other aluminum substrates were either contaminated or used as received. The surface energies of the substrates were determined by contact angle measurements or by using inks with known surface tension. Also a simple wettability test was used. Silicone rubber was then moulded onto the freshly prepared substrates. The silicone rubber adhesion was then evaluated by lap-shear testing, before and after ageing. The failure modes were then characterized by optical microscopy and scanning electron microscopy. 
Based on the results, a minimum requirement for surface cleanliness in an industrial process was given.

\section{EXPERIMENTAL}

\section{Substrate preparation}

Aluminum (AlSi1MgMn) substrates $(150 \times 20 \times 2 \mathrm{~mm})$ were used. Three different types of cleaning methods were evaluated, and compared with a reference and two types of contamination. The cleaning methods and types of pollution were chosen to give a wide range in substrate wettability:

"Clean EtOH": The substrates were rinsed in ethanol (99.9\% purity) in an ultrasonic bath (Bransonic 32) for 10 minutes at ambient temperature. After drying in air, the substrates were primed.

"Clean EtOH/acetone": The substrates were rinsed using ethanol (99.9\% purity) in an ultrasonic bath for 10 minutes at ambient temperature. They were then also rinsed in acetone (99.5\% purity) in the ultrasonic bath for 10 minutes at ambient temperature. After drying in air, the substrates were primed.

"Clean alkaline": The substrates were immersed in an alkaline detergent in an ultrasonic bath for 5 minutes at $60{ }^{\circ} \mathrm{C}$, and then carefully rinsed with deionized water. The detergent was based on alcohol etoxylate and was diluted 10 times in deionized water before use. The $\mathrm{pH}$ of the diluted detergent was 9.7 .

"Contaminated paraffin" and "Contaminated silicone": The substrates were rinsed using ethanol (99.9\% purity) in an ultrasonic bath during 10 minutes at ambient temperature and then dried in air. Contamination, either in the form of paraffin or silicone oil (polydimethylsiloxane, $\mathrm{M}_{\mathrm{n}}$ : $10000 \mathrm{~g} \mathrm{~mol}^{-1}$ ), was then applied using Cleenex tissues. After applying the contamination the substrates were placed in a hot-air oven at $160{ }^{\circ} \mathrm{C}$ for $4 \mathrm{~h}$. Care was taken in order to avoid cross-contamination between samples. 


\section{Primer Application}

A commercial primer, based on a mixture of tetrapropylestersilic acid, tetrakis (2-butoxyethyl) ortosilicate and titanium tetrabutanate dissolved in a volatile cyclic siloxane, was used. The surfaces were primed directly $(<15$ min.) after the sample preparation described in previous section. The substrates were dipped into the primer for $1 \mathrm{~min}$. and then stored in vertical position for allowing removal of excess primer. The primer was then activated for $1 \mathrm{~h}$ in a climatic chamber set at $23{ }^{\circ} \mathrm{C}$ and $23 \% \mathrm{RH}$.

\section{Heat treatment}

After completed priming the samples were placed in a hot-air oven at $160{ }^{\circ} \mathrm{C}$ for $8 \mathrm{~h}$, simulating a process step. Care was taken in order to avoid cross-contamination between samples during the heat treatment.

\section{Preparation of Lap-shear samples}

A commercial grade polydimethylsiloxane was used. The material $\left(\rho=1.55 \mathrm{~g} \mathrm{~cm}^{-3}\right)$ contained approximately $50 \mathrm{wt}$. \% surface-treated aluminatrihydrate, a reinforcing silica filler and a ptcatalyst for crosslinking. Prior to use, the silicone was plasticized in a two roll mill using cooled rolls for a few minutes. Aluminum substrates and $4 \mathrm{~g}$ of the silicone were mounted in a mould and then compression moulded using a Schwabentan press at 200 Bar and $145{ }^{\circ} \mathrm{C}$ for 20 minutes. The sample geometry is shown in Figure 1. The thickness of the rubber layer was $2 \mathrm{~mm}$.

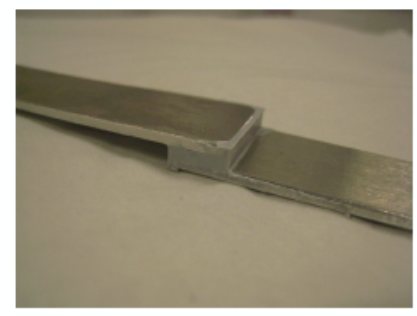

Figure 1. Sample used for Lap-shear test. The silicone rubber (grey color) is moulded between two aluminum substrates. Dimensions of rubber layer: 20 x 10 × 2 mm. 


\section{Lap-Shear Testing}

The test was in accordance with ISO $4587^{16}$ and ISO $10365^{17}$. A $5 \mathrm{kN}$ load cell was used with a pull speed of $10 \mathrm{~mm} \mathrm{~min}^{-1}$. Samples were tested earliest one week after manufacturing in order to allow adhesion between silicone rubber and aluminum substrates to stabilize.

\section{Ageing of substrates}

Ageing was performed by immersion of samples in boiling deionized water during $100 \mathrm{~h}$. The samples were then allowed to dry in desiccators at ambient temperature during at least one week before lap-shear testing.

\section{Contact angle measurements}

Contact angle measurements were performed by the sessile drop technique. Each sample was measured at ten different positions. Deionized water and diiodomethane were used as standard test liquids. The test liquid data is shown in Table 1. The surface energy, calculated as the sum of the polar and dispersive components, was then calculated according to Owens, Wendt, Rabel and Kaelble ${ }^{18,19}$.

Table 1. Total surface tension $\left(\sigma^{\text {tot }}\right)$, as well as polar $\left(\sigma^{\mathrm{p}}\right)$ and dispersive $\left(\sigma^{\mathrm{d}}\right)$ components of water and diiodomethane ${ }^{20}$.

\begin{tabular}{|c|c|c|c|}
\hline Test liquid & $\sigma^{\text {tot }}\left(\mathrm{mN} \mathrm{m}^{-1}\right)$ & $\sigma^{\mathbf{p}}\left(\mathrm{mN} \mathrm{m}^{-1}\right)$ & $\sigma^{\mathrm{d}}\left(\mathrm{mN} \mathrm{m}^{-1}\right)$ \\
\hline Water & 72.8 & 51.0 & 21.8 \\
\hline Diiodmethane & 50.8 & 0.0 & 50.8 \\
\hline
\end{tabular}

As comparison, two tests for characterization of hydrophobicity were used. The first test involved inks ('Dyne Test Pens', Dyne Technology) with known surface tension. Eight different inks with surface tension between 30 to $56 \mathrm{mN} \mathrm{m}^{-1}$ were applied to the substrate surfaces $\left(30,33,36,40,44,48,52\right.$ or $\left.56 \mathrm{mN} \mathrm{m}^{-1}\right)$. The surface energy was determined in the range where one ink wet the surface, whereas another ink with lower surface tension dewets the surface. The other was a "water spray test", according to IEC $62073^{21}$. The surface is first sprayed with water, thereafter the water pattern on the surface, and shape of water droplets, are divided into one of seven different hydrophobicity classes (HC), ranging from 1 to 7 . HC 1 corresponds to a fully hydrophobic surface, whereas HC 7 corresponds to a hydrophilic surface, forming a continuous water film. 


\section{RESULT AND DISCUSSION}

\section{Determination of surface energy or wettability of substrates}

The static contact angles using water and diiodomethane on the different substrates are summarized in Table 2. The substrates appeared more or less hydrophobic. Cleaning with ethanol, or ethanol and acetone resulted in the lowest contact angles for both water and diiodomethane, whereas the contaminated surfaces exhibit contact angles above $90^{\circ}$. The reference exhibited contact angle measurements similar to the contaminated surfaces.

The calculated surface energies, based on the polar and disperse components are listed in Table 3. The spread in surface energy data was estimated by using the standard deviation of the contact angles in Table 2 in the calculations. Surfaces cleaned with the alkaline detergent exhibited the highest surface energy of $56 \mathrm{~mJ} \mathrm{~m}^{-2}$, followed by cleaning using both ethanol and acetone, or ethanol only, exhibiting values of 44 and $38 \mathrm{~mJ} \mathrm{~m}^{-2}$, respectively. The polar component ranged between $8-19 \mathrm{~mJ} \mathrm{~m}^{-2}$ while the dispersive component ranged between 26 $37 \mathrm{~mJ} \mathrm{~m}^{-2}$. The reference, as well as the contaminated surfaces, exhibited a surface energy < $30 \mathrm{~mJ} \mathrm{~m}^{-2}$, of which the polar component was $<2 \mathrm{~mJ} \mathrm{~m}^{-2}$.

Even though the sessile drop technique is accurate, faster and simplified techniques for addressing surface cleanliness was desirable. For industrial applications, the surface energy of large areas can be roughly estimated using test inks or test pens. Liquids with different surface energies are applied to the surface and the wetting pattern is observed. The ink which wet the surface exhibit lower surface tension compared to the surface. If the ink de-wet the surface, the surface tension of the ink is higher than the one of the surface. Pens with surface tension in the range $30-56 \mathrm{mN} \mathrm{m}^{-1}$ were used. The results are summarized in Table 3 . The results showed that the reference and the contaminated surfaces exhibited a surface energy $<33 \mathrm{~mJ}$ $\mathrm{m}^{-2}$, in agreement with the sessile drop measurements. The cleaned surfaces exhibited surface energies $>50 \mathrm{~mJ} \mathrm{~m}^{-2}$, while a value of $28 \mathrm{~mJ} \mathrm{~m}^{-2}$ was obtained by the sessile drop technique. The most probable explanation is that the reference substrate characterized by the sessile drop technique was contaminated from an unknown source. The results of the wettability test (IEC 62073), originally developed for determining the hydrophobicity of electrical outdoor insulation are also summarized in Table 3. The most hydrophilic surfaces (HC 6) were 
cleaned with ethanol or ethanol/acetone. The silicone-contaminated surface was fully hydrophobic, exhibiting HC 1 . The reference substrate exhibited HC 5, which is more similar to the cleaned substrates. This again supports that the low surface energy of the reference, obtained by the sessile drop technique, was not correct.

The atomic surface composition of the cleaned substrates was also characterized by EDX (Table 5). The angle between the surface and the detector was set to $45^{\circ}$ to enhance the surface sensitivity. No significant difference in composition was observed between the reference substrate and substrates cleaned with ethanol or ethanol/acetone, except for the carbon content which was reduced from 2.1 to $1.7-1.9$ wt.\%. The alkaline detergent however, changed the composition significantly. Here the content of carbon and aluminum increased significantly, whereas the amount of oxygen was reduced. This indicates the removal of an oxide layer. However, since the durability of the treatment is poor ${ }^{15}$ and the surfaces were exposed to the ambient air, an new oxide layer may have been gradually formed before priming of the surface.

Table 2. Static contact angle measurements using water or diiodomethane.

\begin{tabular}{lcc}
\hline Substrate type & Contact angle, $\mathbf{H}_{\mathbf{2}} \mathbf{0}$ (deg.) & Contact angle $\mathbf{C H}_{\mathbf{2}} \mathbf{I}_{\mathbf{2}}$ (deg.) \\
\hline Reference & $98 \pm 3$ & $65 \pm 1$ \\
Clean EtOH & $76 \pm 5$ & $57 \pm 2$ \\
Clean EtOH/acetone & $69 \pm 3$ & $49 \pm 3$ \\
Clean surface alkaline & $50 \pm 8$ & $44 \pm 5$ \\
Contaminated paraffin & $94 \pm 2$ & $61 \pm 1$ \\
Contaminated silicone & $103 \pm 2$ & $60 \pm 5$ \\
\hline
\end{tabular}

Table 3. Calculated surface energy $\left(\gamma^{\text {tot }}\right)$ of aluminum substrates, based on the sum of the polar $\left(\gamma^{\mathrm{p}}\right)$ and dispersive $\left(\gamma^{\mathrm{d}}\right)$ components.

\begin{tabular}{|c|c|c|c|}
\hline Substrate type & $\gamma^{\text {tot }}\left(\mathbf{m J} \mathbf{m}^{-2}\right)$ & $\gamma^{P}\left(\mathbf{m J ~ ~ m ^ { - 2 }}\right)$ & $\gamma^{\mathrm{d}}\left(\mathbf{m} \mathbf{J} \mathbf{~ m}^{-2}\right)$ \\
\hline Reference & $27.0 \pm 1.1$ & $1.2+0.7 /-0.5$ & $25.8+0.6 /-0.7$ \\
\hline Clean EtOH & $38.0+3.1 /-3.2$ & $+3.1 /-2.7$ & $30.4+1.0 /-1.1$ \\
\hline Clean EtOH/acetone & $43.9 \pm 3.0$ & $9.3+2.5 /-2.2$ & $34.6 \pm 1.9$ \\
\hline Clean surface alkaline & $56.3 \pm 5.9$ & $18.9+6.0 /-5.4$ & $37.4 \pm 2.7$ \\
\hline Contaminated paraffin & $29.8+0.4 /-0.9$ & $1.5+0.5 /-0.4$ & $28.2+0.7 /-0.6$ \\
\hline Contaminated silicone & $28.7+2.9 /-2.8$ & $+0.4 /-0.2$ & $28.5+3.0 /-2.9$ \\
\hline
\end{tabular}


Table 4. Alternative hydrophobicity tests: hydrophobicity class according to IEC 62073, and by Dyne Pens.

\begin{tabular}{lcc}
\hline Substrate type & Hydrophobicity Class $(\mathbf{H C})$ & Dyne Pen $(\mathbf{m N} / \mathbf{m})$ \\
\hline Reference & 5 & 33 \\
Clean EtOH & 6 & 52 \\
Clean EtOH/acetone & 6 & $52>\mathrm{x}>56$ \\
Clean surface alkaline & 6 & - \\
Contaminated paraffin & 2 & 30 \\
Contaminated silicone & 1 & $\mathrm{x}<30$ \\
\hline
\end{tabular}

$\mathrm{x}=$ surface energy is between or below range of used inks.

Table 5. Atomic surface composition of cleaned aluminum substrates by EDX.

\begin{tabular}{|c|c|c|c|c|c|c|}
\hline \multirow[t]{2}{*}{ Substrate type } & \multicolumn{6}{|c|}{ Wt. $\%$} \\
\hline & $\mathbf{C}$ & $\mathbf{O}$ & Mg & Al & Si & $\mathbf{F e}$ \\
\hline Reference & 2.1 & 10.1 & 4.0 & 81.3 & 1.1 & 1.5 \\
\hline Clean EtOH & 1.9 & 10.1 & 3.3 & 82.8 & 1.0 & 1.0 \\
\hline Clean EtOH/acetone & 1.7 & 10.4 & 4.1 & 81.1 & 1.3 & 1.5 \\
\hline Clean surface alkaline & 3.4 & 5.8 & 1.0 & 87.0 & 0.9 & 2.1 \\
\hline
\end{tabular}

\section{Determination of interfacial strength}

The mechanical strength of the silicone rubber/ aluminum interfaces were evaluated by lap shear testing. The results are shown in Table 6. As expected the presence of contamination, here in the form of paraffin or silicone oil, was detrimental to adhesion. The shear strength was only 0.5 and $0.2 \mathrm{MPa}$, respectively. Without prior surface cleaning the maximum strength was approximately $2 \mathrm{MPa}$. Cleaning the surfaces with ethanol did not improve the strength significantly, whereas the combination of ethanol and acetone resulted in a significant increase in shear strength up to $2.5 \mathrm{MPa}$. The high strength of the reference substrate showed that the ink test and the wettability test showed more correct values, compared to the sessile drop technique. Cleaning with the alkaline solution resulted in a shear strength of $2.2 \mathrm{MPa}$. Since the use of the alkaline detergent resulted in similar shear strength as for ethanol and acetone, it is believed that the mechanical properties of the aluminum oxide layer were not a critical factor in this experiment. If a weak oxide layer would have been present, and if this layer was successfully removed by the alkaline detergent, those samples would have exhibited a higher interfacial strength.

It is also important to make an estimation of the long-term stability of the interfacial strength ${ }^{10}$. In the current work this was addressed using a $100 \mathrm{~h}$ water boiling test. What is tested here is the hydrolytic stability of the coupling agent and the silicone rubber in the interface. The 
siloxane bonds between the coupling agent and the aluminum substrate are reversible. If water can get to the siloxane bond, hydrolysis may occur, weakening the interface. There is, however, also a probability that the equilibrium is shifted back to the siloxane bond while removing the water (when stored in an dry environment after water immersion) ${ }^{3}$ restoring the interfacial strength.

Table 6. Lap-shear testing of silicone rubber interfaces before and after ageing.

\begin{tabular}{lcc}
\hline \multicolumn{1}{c}{ Substrate type } & Shear strength (MPa) & Shear strength, aged (MPa) \\
\hline Reference & $1.98 \pm 0.25$ & $1.40 \pm 0.42$ \\
Clean EtOH & $1.93 \pm 0.35$ & $1.62 \pm 0.36$ \\
Clean EtOH/acetone & $2.46 \pm 0.17$ & $2.20 \pm 0.08$ \\
Clean surface alkaline & $2.22 \pm 0.21$ & $2.49 \pm 0.11$ \\
Contaminated paraffin & $0.47 \pm 0.18$ & $0.67 \pm 0.20$ \\
Contaminated silicone & $0.20 \pm 0.09$ & $0.60 \pm 0.87$ \\
\hline
\end{tabular}

The shear strength is plotted against the total surface energy in Figure 2a. The scatter in data is significant. It can however be concluded that the shear strength increased with increasing surface energy of the substrate. In addition, a surface energy above $45 \mathrm{~mJ} \mathrm{~m}^{-2}$ was required to obtain a stable interface, even after the water immersion test. The reference substrates exhibited a high initial strength; however after ageing the strength was significantly reduced. This indicated an irreversible hydrolysis of the interface. A slight reduction was also observed after cleaning with ethanol only. Cleaning with ethanol and acetone, or the alkaline detergent, resulted in the highest initial strength. These interfaces were moreover not significantly influenced by the ageing test, indicating good long-term properties.

The importance of the polar component can be seen in Figure 2b, where the shear strength increases rapidly if the polar component is $>2 \mathrm{~mJ} \mathrm{~m}^{-2}$. If the polar component was between 2 $-10 \mathrm{~mJ} \mathrm{~m} \mathrm{~m}^{-2}$, the initial strength appeared high, around $2 \mathrm{MPa}$; but was significantly reduced after ageing, in combination with a large scatter in data. This indicated a region where the quality of adhesion was not stable, which is undesirable from a process point of view. This also indicates a poor long-term stability of the interfacial strength. Finally if the polar component was $>10 \mathrm{~mJ} \mathrm{~m}^{-2}$, a stable interface was obtained also after the ageing test, in combination with a lower scatter in data. This indicates a stable process, in combination with good long-term properties. The influence of the dispersive component is less clear, as can be seen in Fig. 2c. The highest shear strength was obtained if this component was $>30 \mathrm{~mJ} \mathrm{~m}^{-2}$. 
A few samples contaminated with silicone oil increased in shear strength after ageing, resulting in a standard deviation larger than the average value. It is suggested that this was caused by a partial hydrolysis of the silicone oil during water immersion at elevated temperature, forming additional silanol groups. These then condensed into Si-O Si bonds upon drying, thereby strengthening the interface. The linear structure and relatively low molar mass of the silicone oil may also have allowed interpenetration into the silicone rubber network, enhancing the interfacial strength ${ }^{22}$.

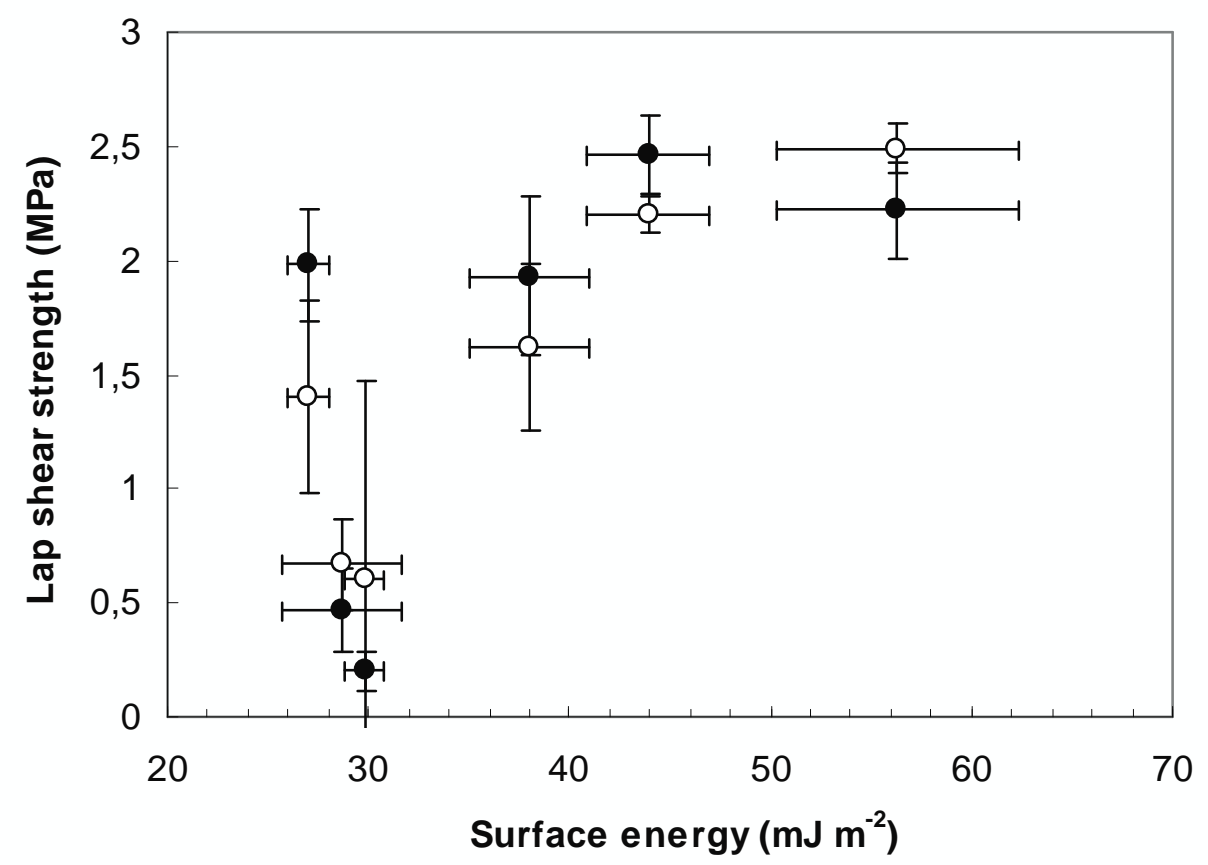

Figure 2 a). Shear strength of silicone joints before $(\bullet)$ and after (०) ageing vs. surface energy of the aluminum substrates prior to priming. 


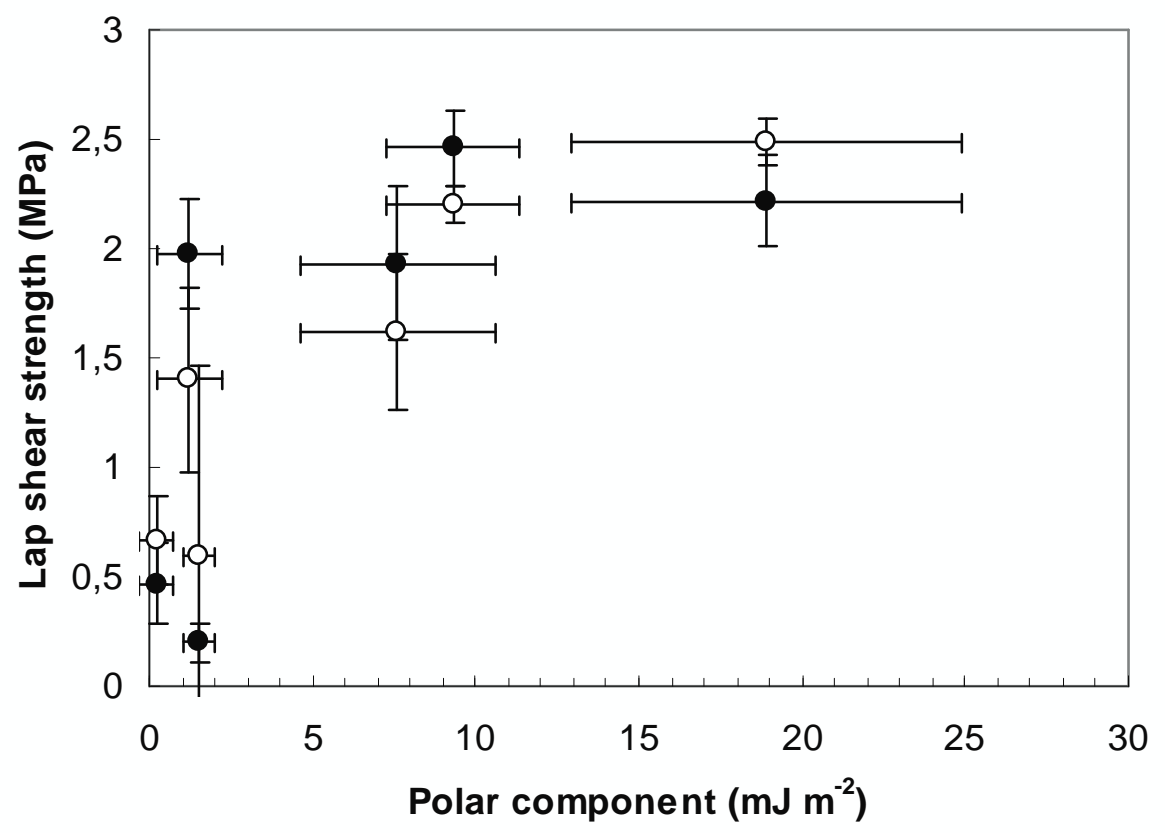

Figure $2 \mathrm{~b}$ ). Shear strength of silicone joints before $(\bullet)$ and after $(\circ)$ ageing vs. the polar component of the aluminum substrates prior to priming.

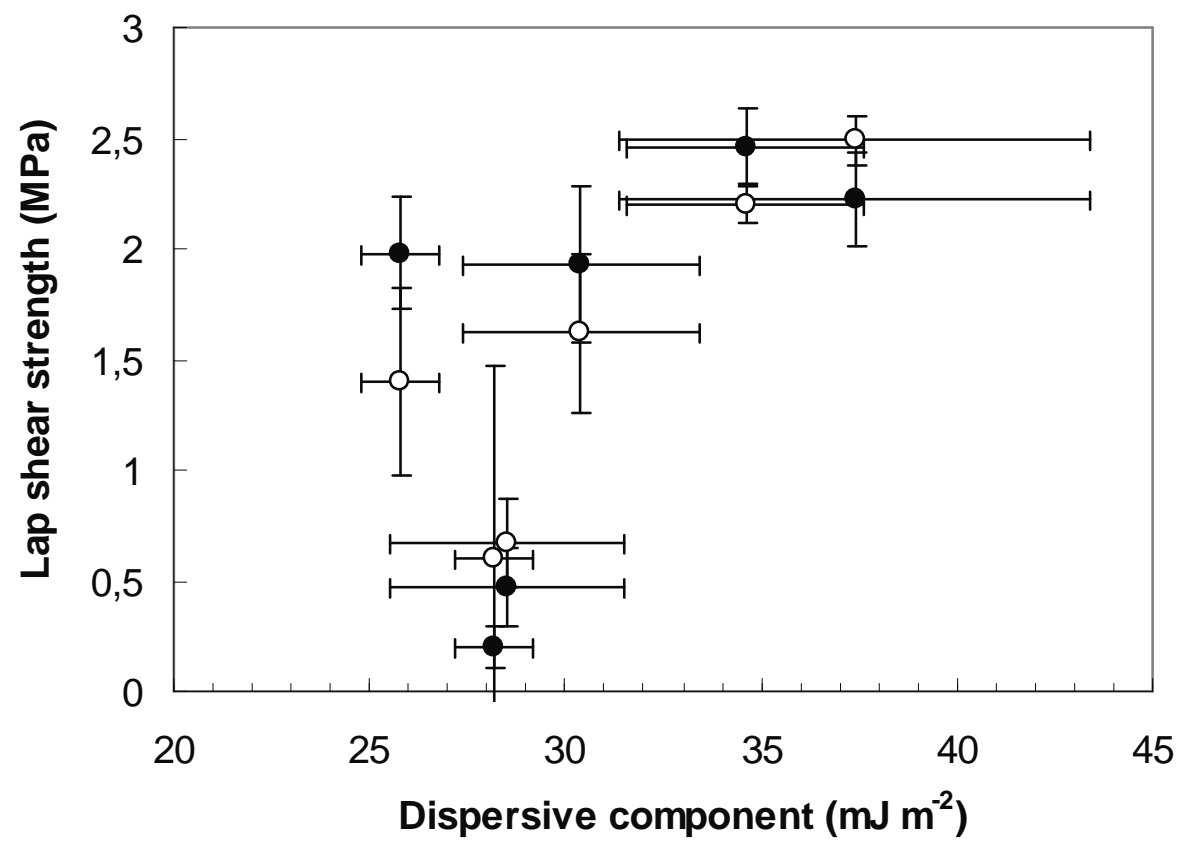

Figure $2 \mathrm{c})$. Shear strength of silicone joints before $(\bullet)$ and after $(\circ)$ ageing vs. the dispersive component of the aluminum substrates prior to priming. 


\section{Classification of interfacial failure modes}

The fractured surfaces were investigated by optical microscopy and scanning electron microscopy. The failures were divided into three different modes: 1) cohesive failure, 2) cohesive-interfacial failure and 3) adhesive failure. In the cohesive failure the fracture occurred within the rubber, leaving residues on both substrate surfaces. This showed that the interfacial strength was higher than the strength of the rubber. In a cohesive-interfacial failure the fracture occurred both in the rubber, between primer and oxide layer and within the oxide layer (See Fig. 3). During SEM analysis, Energy Dispersive X-ray (EDX) analysis was performed to map the lateral distribution of relevant elements of this failure mode. The SEM image is shown in Fig 4a, whereas the content of C, O, Al, Si and Ti are mapped in Fig. 4 b-d, respectively. Since the primer contained $\mathrm{Ti}$, it can be concluded that the fracture occurred either within the aluminum oxide layer (containing $\mathrm{Al}$ and $\mathrm{O}$ only) or within the interface primer/rubber (containing $\mathrm{Si}, \mathrm{C}, \mathrm{O}$, and $\mathrm{Ti}$ ). The adhesive failure occurred between primer and substrate surface. These surfaces appeared shiny in the optical microscope, and did not contain any residues of rubber (Fig. 5). In addition, no presence of Ti could be detected on the aluminum substrates by EDX. In these samples, it is believed the primer did not bond completely to the substrate surfaces. Thus EDX was a helpful tool in determining the failure modes for identifying the position of the primer, if it contains a specific element.

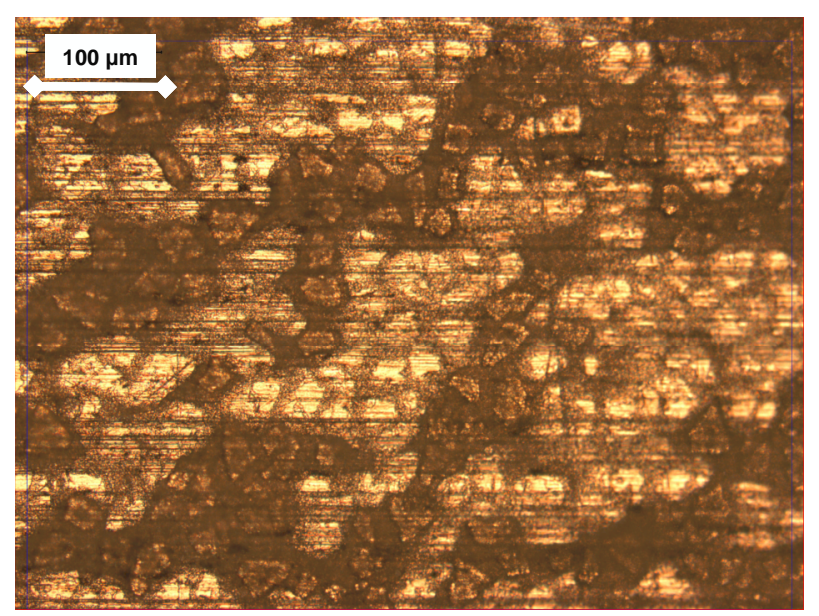

Figure 3. Optical micrograph of an aluminum substrate after a cohesive-interfacial failure.

The bright areas consist of aluminum, whereas the dark areas consist of silicone rubber. 


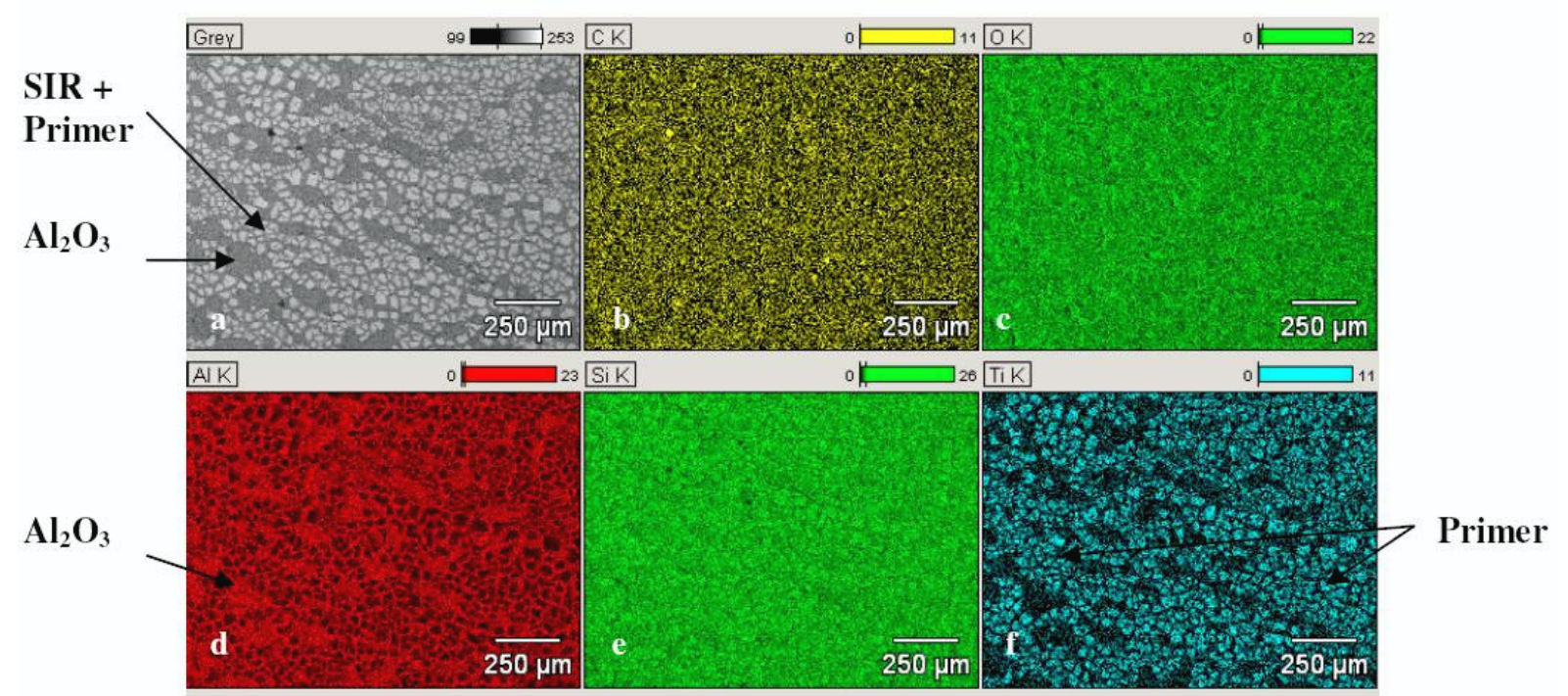

Figure 4. SEM/EDX image of an aluminum substrate after a cohesive-interfacial failure. (a)

SEM image and EDX element maps of (b) Carbon, (c) Oxygen, (d) Aluminum, (e) Silicon, (f) Titanium.

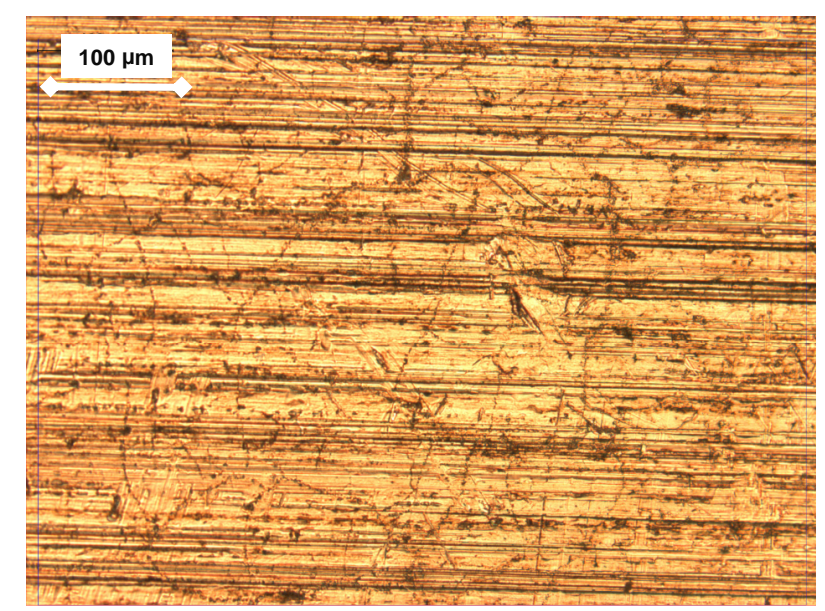

Figure 5. Optical micrograph showing an aluminum surface after an adhesive failure.

The different types of failures, before and after ageing are summarized in Table 7. Even though only 5-6 samples of each type were investigated, it can be concluded that the adhesive failures correlated with the weakest interfaces. This relation between surface energy and amount of adhesive failures is more clearly shown in Figure 6. In order to avoid adhesive failures, a surface energy $>45 \mathrm{~mJ} \mathrm{~m}^{-2}$ was desirable, Comparing with the simplified wettability test (IEC 62073), this would correspond to $\mathrm{HC} \geq 6$. This is also in rough agreement with the ink test, which indicated that the surface energy should be at least $52 \mathrm{~mJ} \mathrm{~m}^{-2}$. Thus in order to make quick control of the surface cleanliness of incoming parts before injection moulding of silicone rubber onto aluminum substrates, either of the two simplified test 
methods could give sufficient information. It should however be kept in mind that none of the evaluated methods can differ between mechanically strong or weak oxide layers.

Table 7. Summary of types of failure modes after lap shear testing, before and after water immersion (ageing).

\begin{tabular}{lccc}
\hline Substrate type & Cohesive & No of Lap Shear Failures (Virgin/ Aged) \\
& $2 /-$ & $-/ 1$ & Cohesive-interfacial \\
\hline Reference & $2 / 1$ & $1 / 1$ & $4 / 4$ \\
Clean EtOH & $2 /-$ & $4 / 5$ & $3 / 3$ \\
Clean EtOH/acetone & $-/-$ & $5 / 5$ & $-/ 1$ \\
Clean surface alkaline & - & - & $-/-$ \\
Contaminated paraffin & - & - & $6 / 6$ \\
Contaminated silicone & & & $6 / 6$ \\
\hline
\end{tabular}

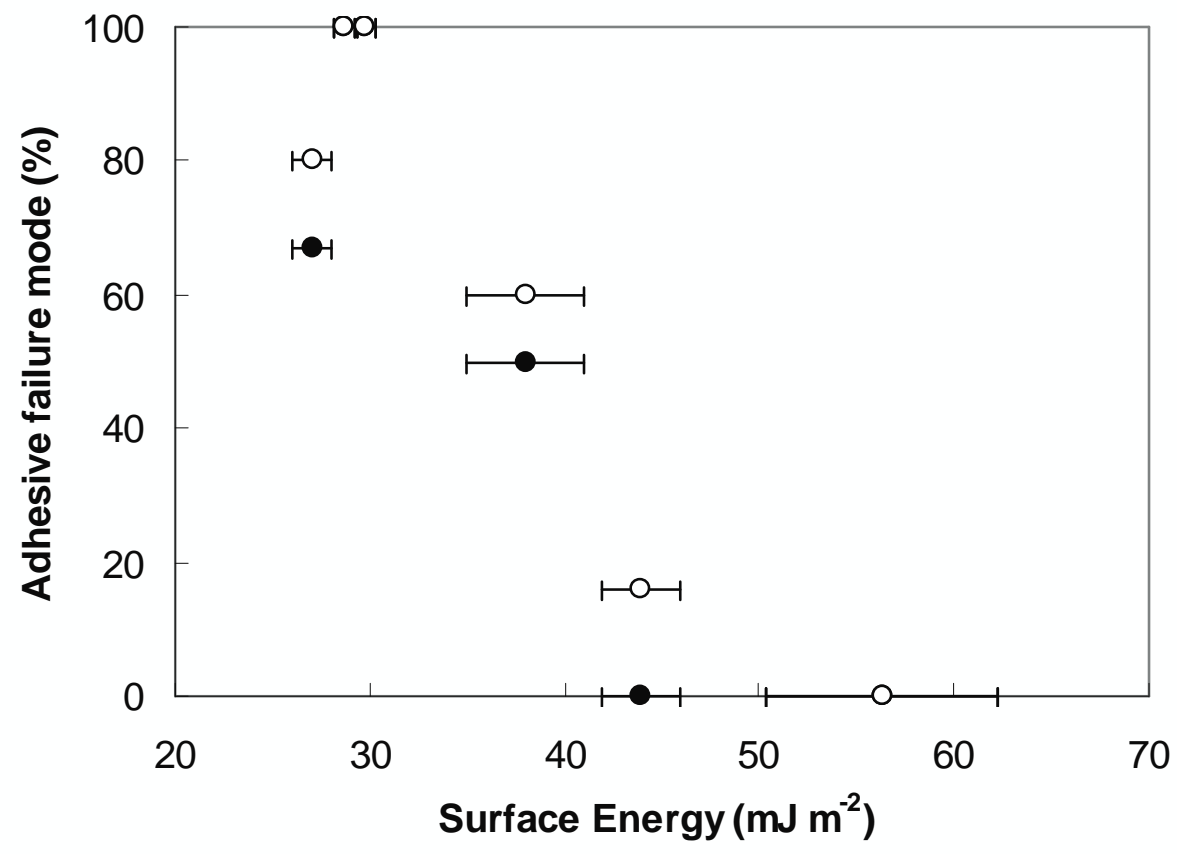

Figure 6. Per centage of adhesive failures of silicone joints before $(\bullet)$ and after $(\circ)$ ageing vs. surface energy of the aluminum substrates prior to priming. 


\section{CONCLUSIONS}

The required 'cleanliness' of aluminum substrates for obtaining good and reproducible adhesion to silicone rubber was investigated. Aluminum substrates were prepared, ranging from 'contaminated' to different degrees of 'cleaned'. The surface energy of the substrates was determined by contact angle measurements and the atomic surface composition was characterized by Energy dispersive X-ray (EDX). The surfaces were also compared using simplified characterization methods, such as a wettability test (IEC 62073) or by the use of inks with known surface tension. After silicone rubber moulding onto the cleaned and primed substrates, the strength of the rubber/ aluminum interfaces was evaluated by lap-shear testing. Lap shear testing was performed before and after ageing by immersion in boiling water during 100h. The failure modes after lap shear testing were determined using optical and scanning electron microscopy and divided into three different categories: cohesive failure, adhesive failure or a mixture thereof. EDX mapping was useful in clarifying the failure modes by determining the position of the primer, which contained Ti. This element was not present in the substrate or the silicone rubber. It was concluded that in order to obtain a strong and stable interface between aluminum substrates and silicone rubber, the surface energy of the substrate before priming should be $>45 \mathrm{~mJ} \mathrm{~m}^{-2}$, including a polar component of $>10 \mathrm{~mJ} \mathrm{~m}^{-2}$. This corresponded to a hydrophobicity class $\geq 6$, according to IEC 62073 . 


\section{REFERENCES}

${ }^{1}$ Noll W 1968 Chemistry and Technology of Silicones (Academic Press, New York)

${ }^{2}$ Clarson S J, Semlyen, J A 1993 Siloxane Polymers (PTR Prentice Hall, New Jersey)

${ }^{3}$ Pludderman, E P 1991 Silane Coupling Agents, Second Edition (Plenum Press, New York)

${ }^{4}$ Kim E S, Kim H S, Jung S H, Yoon J S 2006 Adhesion properties and thermal degradation of silicone rubber J. Appl. Poly. Sci. 103 2782-2787

${ }^{5}$ Morent R, De Geyter N, Axisa F, De Smet N, Gengembre L, De Leersnyder E, Leys C, Vanfleteren J, Rymarczyk-Machal M, Schacht E, Payen E 2007 Adhesion enhancement by a dielectric barrier discharge of PDMS used for flexible and stretchable electronics J. Phys. $D$ Appl. Phys. 40 7392-7401

${ }^{6}$ Garbassi F, Morra M, Occhiello E 1994 Polymer Surfaces. From Physics to Technology (J. Wiley \& Sons Ltd, West Sussex, England).

${ }^{7}$ Goldberg H D, Cha G S, Brown R B 1991 A quantitative adhesion test for thin polymer films on silicon substrates J. Appl. Poly Sci. 43 1287-1292

${ }^{8}$ Iqbal H M S, Bhowmik S, Benedictus R 2010 Surface modification of high performance polymers by atmospheric pressure plasma and failure mechanism of adhesive bonded joints Int. J. Adhesion \& Adhesives 30 418-424

${ }^{9}$ Iqbal H M S, Bhowmik S, Benedictus R 2010 Effect of plasma treatment and electron beam radiations on the strength of nanofilled adhesive-bonded joints Polym. Eng. Sci. 50 1505-1511

${ }^{10}$ Dartevelle C, McAlpine, Thomphson G E, Alexander M R 2003 Low pressure plasma treatment for improving the strength and durability of adhesively bonded aluminum joints Surface and Coatings Techn. 173 249-258

${ }^{11}$ Petrie E M 2000 Handbook of Adhesives and Sealants (McGraw-Hill, New York) 151-155

${ }^{12}$ MIG WELD GmbH International Wattstrasse 2 D-94405 Landau/Isarnfo, http://www.migweld.de/service/schweissen-von-Aluminum/oxidschicht.html 
${ }^{13}$ Hinüber H, Hufnagel W, Alluminium-Zentrale, Düsseldorf, http://www.bkalu.de/html/body_oberflaechen.html

${ }^{14}$ Prolonga S G, Urena A 2009 Effect of surface pre-treatment on the adhesive strength of epoxy-aluminum joints Int. J. Adhesion \& Adhesives 29 23-31

${ }^{15}$ Critchlow G W, Brewis D M 1996 Review of surface pretreatments for aluminum alloys Int. J. Adhesion and Adhesives 16 255-275

${ }^{16}$ ISO 4587: 2003 Adhesives - Determination of tensile lap-shear strength of rigid-to-rigid bonded assemblies

${ }^{17}$ ISO 10365: 1992 Adhesives - Designation of main failure patterns

${ }^{18}$ Owens D K, Wendt RC 1969 Estimation of the surface free energy of polymers J. Appl. Polym. Sci. 13 1741-1747

${ }^{19}$ Kaelble D H 1970 Dispersion-Polar Surface Tension Properties of Organic Solids $J$. Adhesion 2 66-81

${ }^{20}$ Ström G, Frederiksson M, Stenius P 1987 Work of adhesion, and interfacial tensions at a dissolving hydrocarbon surface J. Coll. Interf. Sci. 119 352-361

${ }^{21}$ IEC TS 62073: 2003 Guidance on the measurement of wettability of insulator surfaces

${ }^{22}$ Gedde U W, Hellebuych A, Hedenqvist M 1996 Sorption of low molar mass silicones in silicone elastomers Polym. Eng. Sci. 3 2087-2082 


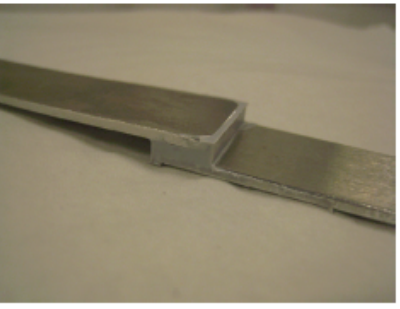

Petersson et al, Figure 1

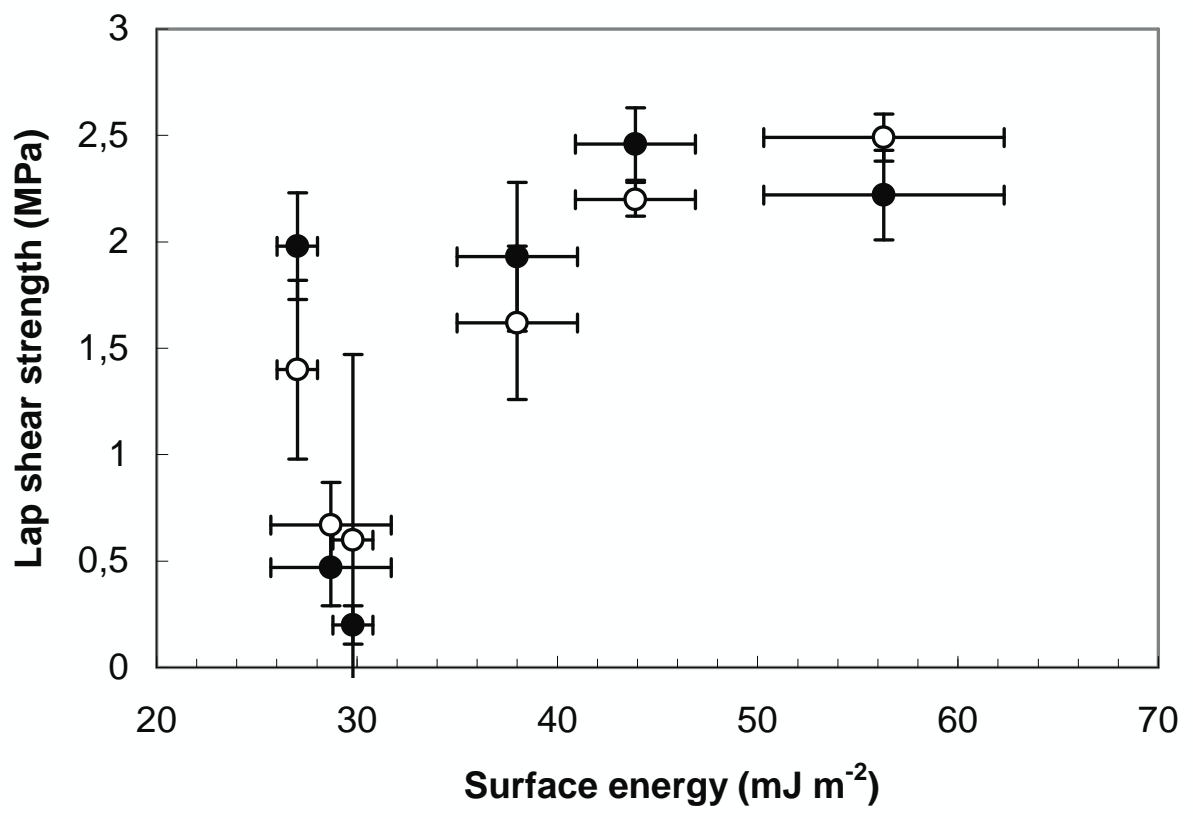

Petersson et al, Figure 2a 


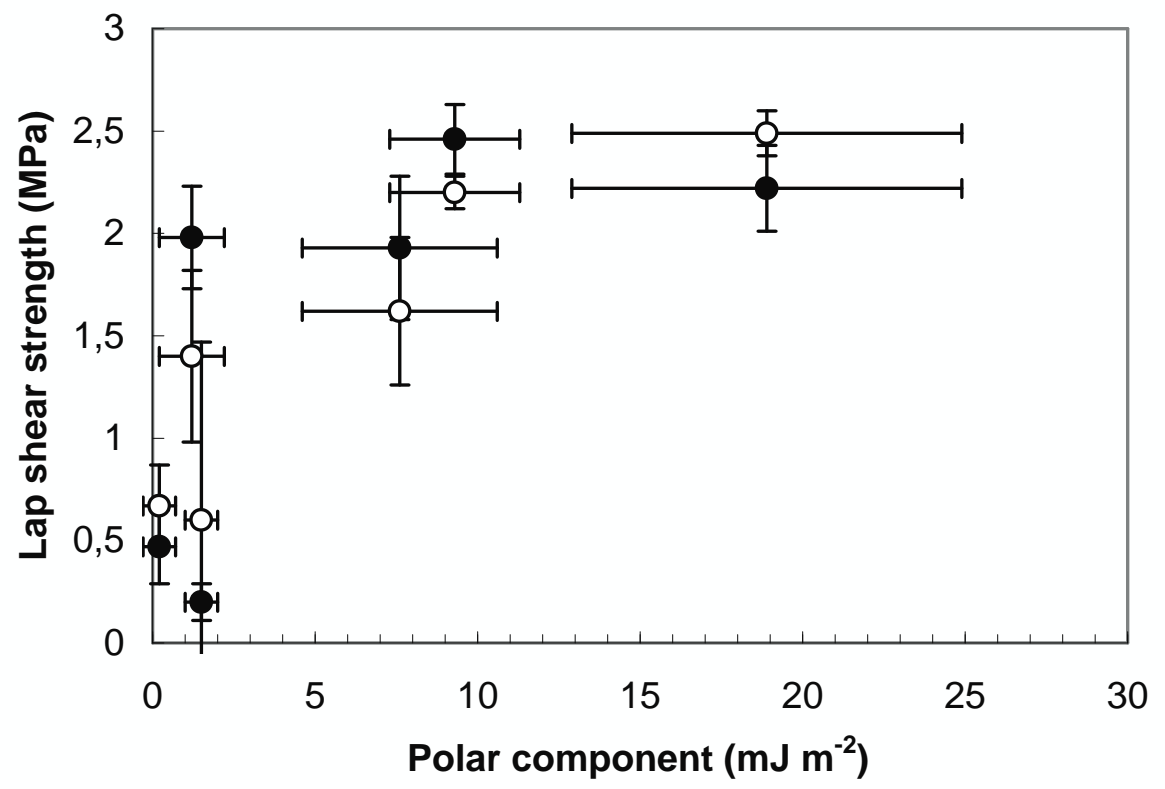

Petersson et al, Figure $2 b$

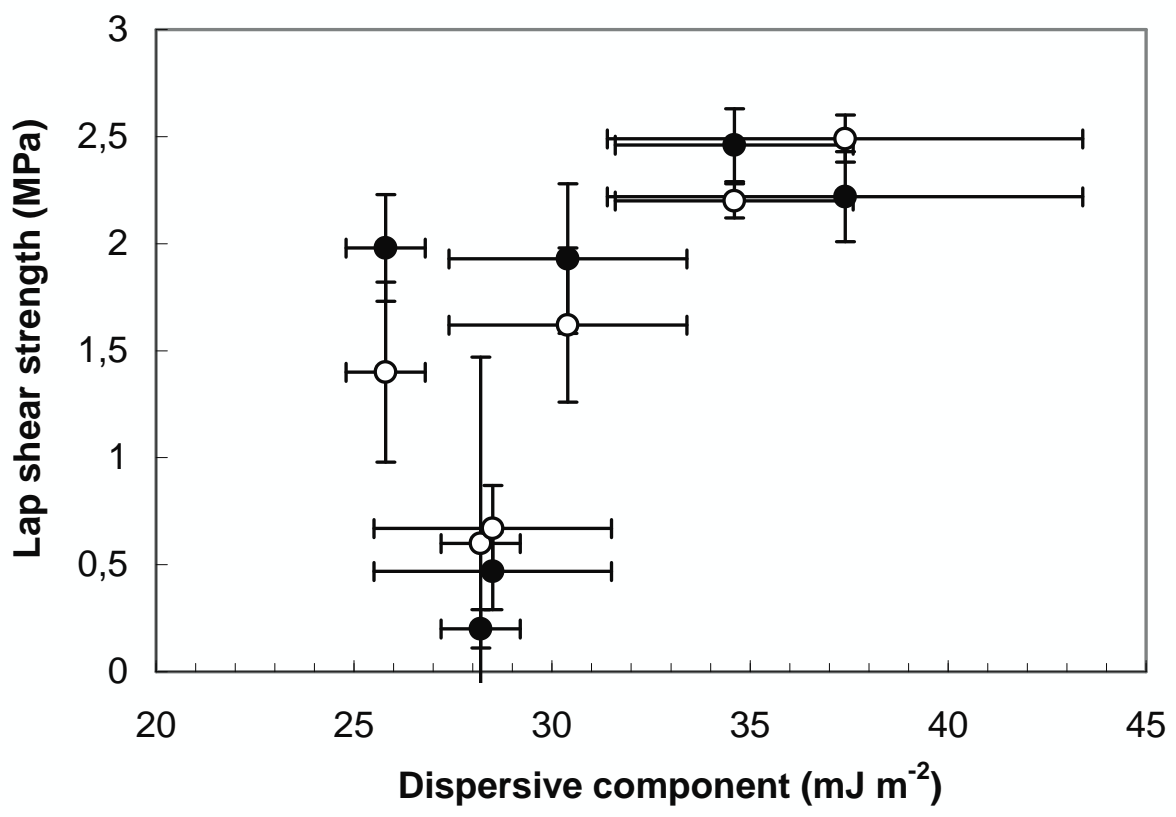

Petersson et al, Figure 2c 


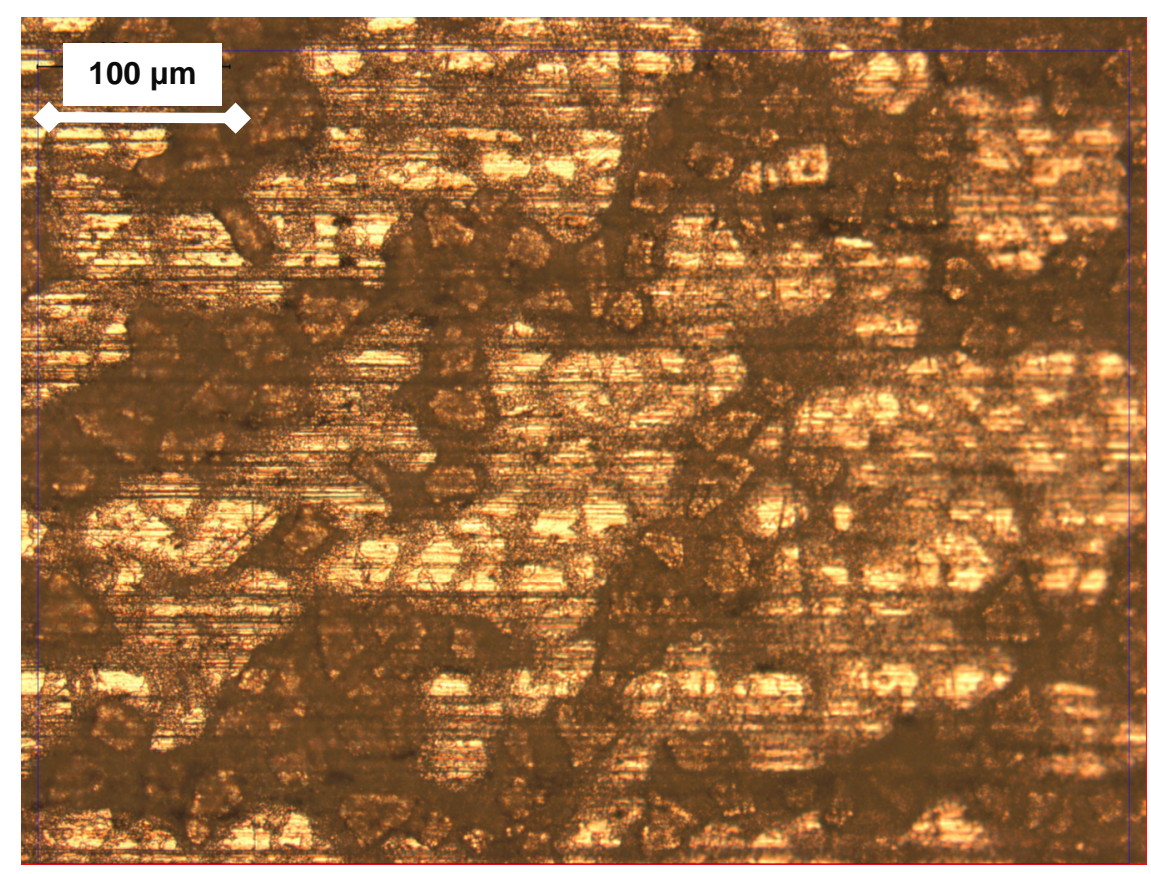

Petersson et al, Figure 3

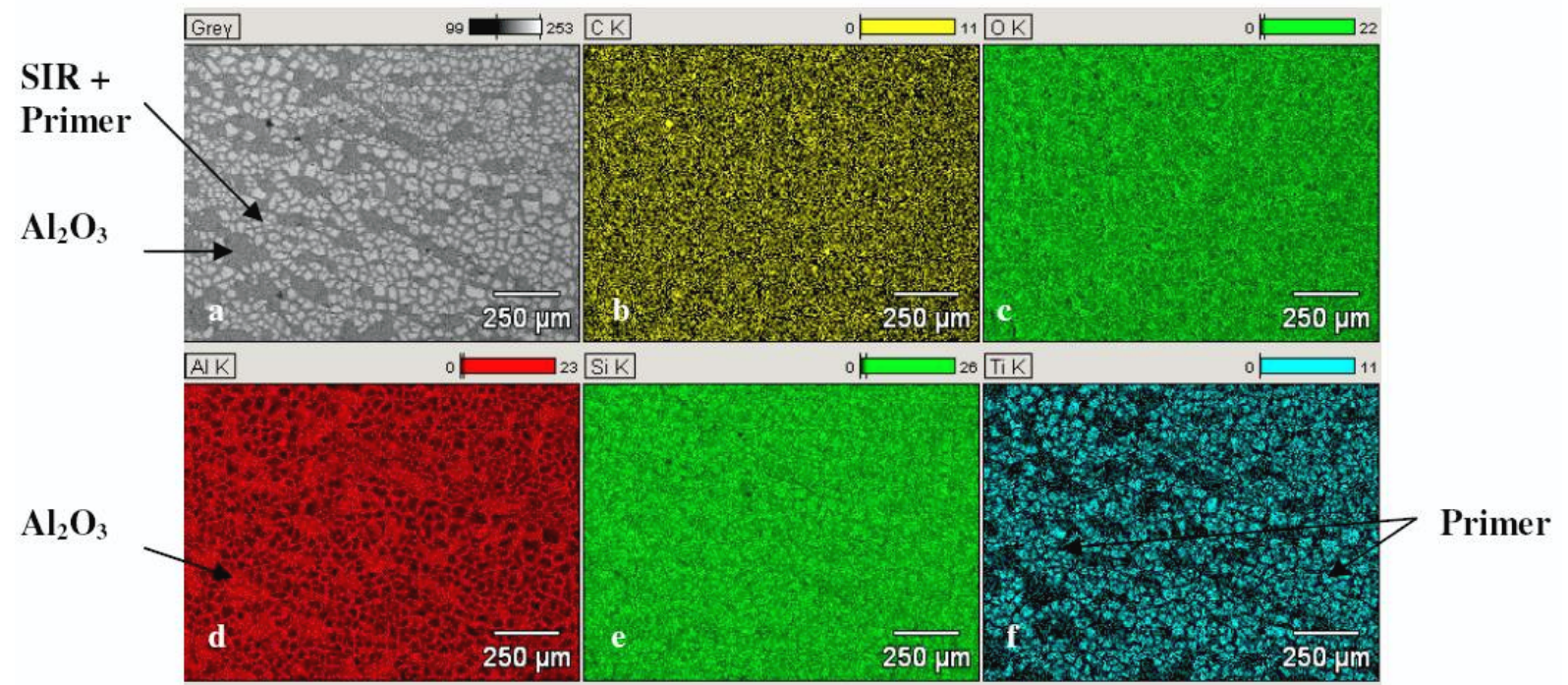

Petersson et al, Figure 4 


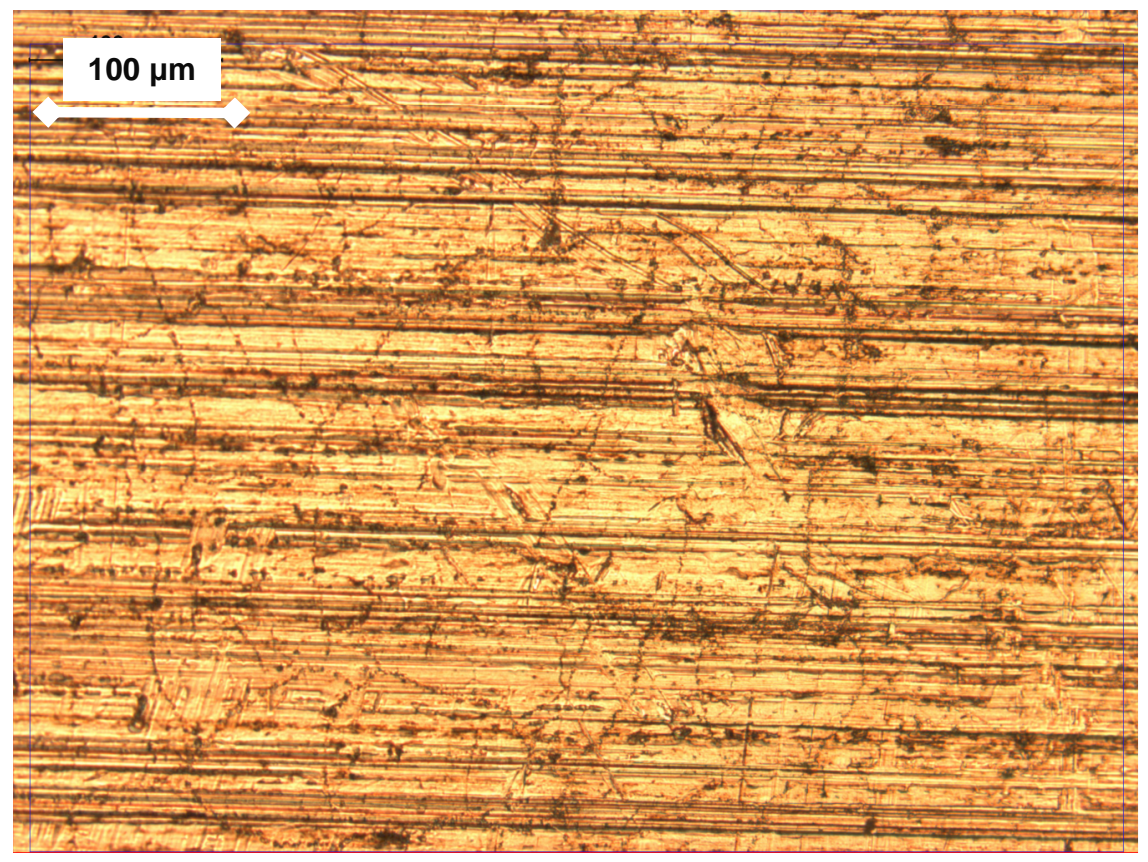

Petersson et al, Figure 5

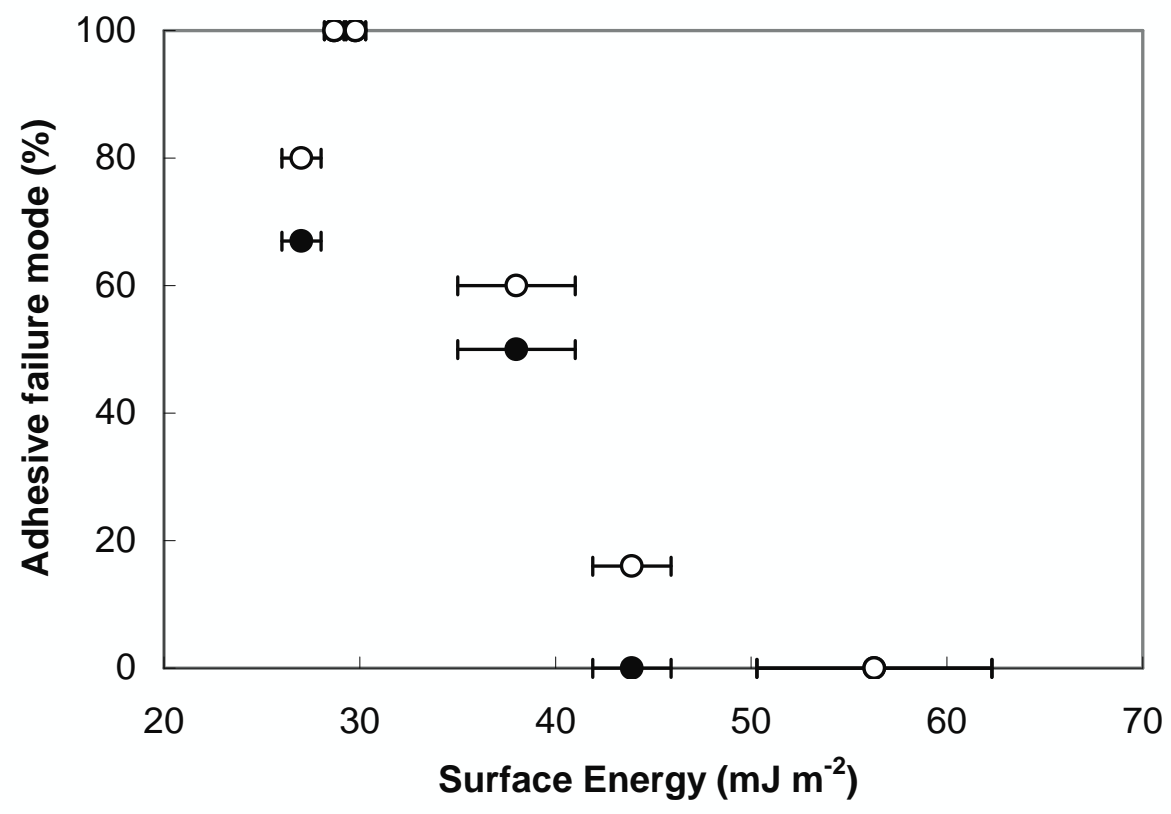

Petersson et al, Figure 6 